\title{
Zur Definition von Public Relations
}

Klaus Merten

Public Relations als Wissenschaft befinden sich noch immer in einem vorläufigen Stadium, was deutlich an den mebr als 500 Definitionsversuchen, abzulesen ist. An diesen Befund knüpft der vorliegende Beitrag an: Er fragt in einem ersten Anlauf zunächst nach den Schwierigkeiten bei der Definitionsbildung und versucht, aus vorliegenden Definitionen wesentliche Aussagen zu extrabieren, die auf ein breiteres Verständnis von PR verweisen. In einem zweiten Anlauf werden Bezüge zu Kommunikation und deren Management hergestellt. In einem dritten Anlauf werden Vergleiche von Leistungen (Konfektion vs. Unikat) und Leistungskriterien (Wabrheit versus Unwabrheit) zwischen Non-PR und $P R$ bergestellt. Auf der Basis dieser Befunde wird in einem letzten Schritt in differentialistischer Perspektive eine notwendig abstraktere Definition vorgestellt: Public Relations sind das Differenzmanagement zwischen Fakt und Fiktion durch Kommunikation über Kommunikation in zeitlicher, sachlicher und sozialer Perspektive.

Schlagwörter: Public Relations, Definition, PR als Wissenschaft, Wahrheit, Management, Differenztheorie

Public Relations haben sich, wie alle Wissenschaften, aus praktischen Problemen heraus entwickelt, mit zunehmender Reife ihren Gegenstandsbereich ständig erweitert und dabei Komplexität und Anwendungstiefe ihrer Theorien und Methoden konkordant gesteigert. Gleichwohl fehlt bis heute - was in so gut wie in allen Lehrbüchern zu Public Relations bemängelt wird - eine konsentierte Definition: „Public Relations has struggled with an identity crisis and has failed to adopt an accepted definition of what it is nor agreed to what it does“ (L'Etang/Pieczka 2006: 90; Fröhlich 2005; Kunczik 2002: 23ff.; Avenarius 1995: 51ff.; Cutlip et al. 1994: 1ff.; Haedrich et al. 1982: 188).

Zum einen fehlt der definitive Bezug auf ein konsentiertes Erkenntnisobjekt - was für evolvierende Wissenschaften allerdings nicht ungewöhnlich ist. ${ }^{1}$ Zum zweiten haben sich Public Relations aus praktischem Handeln entwickelt, das als solches zunächst nicht begründungs-, sondern allenfalls erklärungsbedürftig erscheint und noch wenig Interesse an Abstraktion zeigt (vgl. Fröhlich 2005: 95ff.). Erschwerend kommt hinzu, dass die einschlägigen Standesorganisationen aus einsichtigen Gründen in ihre PR-Definition gern eine positiv getönte ethische Zierleiste einarbeiten, die diese dann im strengen Sinne der Definitionslehre unbrauchbar macht. Viertens gelten Public Relations im Verständnis der noch jungen Kommunikationswissenschaft als eine oft riskante Anwendung gelegentlich riskanter kommunikativer Techniken, deren wissenschaftliche Beobachtung daher eher gescheut wird. Insgesamt hat dies dazu geführt, dass die wis-

1 Wilmont Haacke (1957: 129) sah Public Relations noch als, Heimsuchung der Deutschen Publizistik'; Pavlik (1987: 16) folgt ihm darin mit der Umschreibung des Wesens von PR als „The nature of the beast“ und in dem von Noelle-Neumann et al. (1989ff.) herausgegebenen Lexikon Publizistik/Massenkommunikation tauchen als Stichwortartikel bis 1999 weder ,Public Relations' noch ,Öffentlichkeitsarbeit' auf. Dazu passt schließlich auch das Stereotyp, die Vertreter dieser Branche beharrlich als, Sektglashalter mit Zahnpastalächeln, an Stresemann abgelöscht' oder als ,PR-Fuzzies' zu identifizieren. Doch auch das ist keinesfalls ungewöhnlich, denn für den Beruf des Journalisten gab es in der Anfangszeit ähnlich bösartige Sentenzen (vgl. Stieler 1969: 30ff.). 
senschaftliche Definition des Begriffes Public Relations wenig fruchtbar geblieben ist oder sogar abgelehnt wird. ${ }^{2}$ Ja, es scheint fast einfacher zu beschreiben, was Public Relations nicht ist als das, was genau Public Relations darstellt (vgl. so Scharf 1971: 166).

An diesen Befund knüpft der vorliegende Beitrag an: Er fragt in einem ersten Anlauf zunächst nach den Schwierigkeiten bei der Definitionsbildung und versucht, aus vorliegenden Definitionen wesentliche Aussagen zu extrahieren, die auf ein breiteres Verständnis von PR verweisen. In einem zweiten Anlauf werden Bezüge zu Kommunikation und deren Management hergestellt. In einem dritten Anlauf werden Vergleiche von Leistungen (Konfektion vs. Unikat) und Leistungskriterien (Wahrheit versus Unwahrheit) zwischen Non-PR und PR hergestellt. Auf der Basis dieser Befunde wird in einem letzten Schritt eine notwendig abstraktere Definition vorgestellt und diskutiert.

\section{Probleme bei der Definition von PR}

Schwierigkeiten, Public Relations zu definieren, gibt es eine ganze Reihe (vgl. Fröhlich 1995). Die wichtigsten fünf scheinen zu sein:

1) Definitionen von PR sind vielfach perspektivisch und folgen unterschiedlichen Interessen.

2) Der Begriff Public Relations stellt kein Objekt, sondern eine Relation dar: Zwar stellt auch ein Kommunikationsprozess eine Relation dar, aber dessen Endpunkte sind in der Regel (Ausnahme: Gerüchte) klar identifizierbare Kommunikanten, und die Beziehung kann z. B. durch identifizierbare und analysierbare Kommunikationsangebote (Inhalte) präzisiert werden. Bei Public Relations ist der Adressat stets eine eher diffuse Adresse, nämlich die Öffentlichkeit, die in der Regel durch den Begriff der Zielgruppe substituiert wird. ${ }^{3}$

3) Die Struktur der dabei zugrunde liegenden Kommunikation ist diffus: PR beruht expressis verbis auf einer Vielzahl eingesetzter, aber stark heterogener, aufeinander bezogener und auf Kontinuität zielender Kommunikationsprozesse, deren kalkulierte summarische Wirkung die jeweiligen damit zu erzielenden Beziehungen auch erzeugen soll.

4) Das Verständnis von Public Relations wandelt sich mit der Entwicklung der Medien: Schon Scharf (1971) und Cutlip et al. (1994: 2ff.) zeigen für amerikanische Verhältnisse, wie schnell und wie durchgängig sich PR verändern - von der schieren Propaganda (publicity) 1894 über ein „Engineering of Consent“ (Bernay 1947) bis hin zu einer

2 Nur als Indiz: 1992 veröffentlichen Avenarius/Armbrecht unter dem Titel „Ist Public Relations eine Wissenschaft?" das Ergebnis einer viel beachteten Konferenz der Quandt-Stiftung, in der 18 Beiträge zur Frage, ob PR eine Wissenschaft sei oder eine solche werden könne, diskutiert werden. Sie bemühen dabei eine Fülle von theoretischen Ansätzen. Doch es findet sich nur eine einzige, sehr allgemeine, funktionale Definition von Botan (1992: 20), nämlich „PR is the use of communication to adapt relationships", die wiederum an die Definition von Grunig und Hunt, verstanden als „management of communication between an organization and its publics“ (Grunig/Hunt 1984: 6), angelehnt ist. Manfred Rühl, einer der Teilnehmer, erklärt zudem mit Nachdruck, er sei geneigt, jedem Studenten, der die Definition von PR in einer Arbeit versuche oder anmahne, dies mittels genügend aussagekräftiger roter Anmerkungen am Seitenrand auszureden (persönliche Mitteilung, Oktober 1999).

3 Eine Zielgruppe ist durch demographische Eigenschaften definiert. Dagegen sind Öffentlichkeiten oder Teilöffentlichkeiten Personen oder Personengruppen mit gemeinsamen Interessen, deren Mitglieder sich nicht kennen müssen, die aber in der Verfolgung ihrer Interessen de facto aufeinander treffen und reale Gestalt annehmen könnten (z. B. bei einer Demonstration). 
„management function that establishes and maintains mutually beneficial relationships between an organization and the publics on whom its success or failure depends" (Cutlip et al. 1994: 1). Gleiches gilt in der Tendenz und mit einem gewissen time-lag auch für deutsche Verhältnisse (vgl. Scharf 1971; Kunczik 2002: 101ff.). Das rührt zum einen daher, dass Public Relations a) starke Berührungsfächen zu anderen Kommunikationsberufen haben, vor allem zum Journalismus und zur Werbung, dass b) mehrere Disziplinen, vor allem das Marketing, die Kommunikationswissenschaft und die Politischen Wissenschaften (vor allem über die Anwendung von Propaganda) Public Relations als ihr Arbeits- und Forschungsfeld reklamieren und dass sich c) das entstebende Berufsfeld PR vom Ende des 19. Jahrhunderts bis heute gerade in einer Zeit entwickelt, wo das Mediensystem evolviert, wo die Entfesselung der Kommunikation abläuft, so dass jedes neue Medium neue Möglichkeiten für Public Relations offeriert, die sofort aufgegriffen werden und zu völlig neuen Anwendungsbereichen führen (derzeit beispielsweise: Online-PR). Damit ergibt sich auf lange Sicht die Notwendigkeit d) bestimmte Teilbereiche neu zu ordnen, was nicht selten disziplinäre Konflikte erzeugt und bestimmte Schulen und den Streit zwischen diesen begünstigt. ${ }^{4}$ Unter diesem Veränderungsdruck ${ }^{5}$ hat es e) die nacheilende Verwissenschaftlichung von Public Relations besonders schwer: Sie agiert auf einem schwankenden Boden, muss ihren Geltungsbereich erst erkämpfen, einen anerkannten Einstellplatz bei einer anerkannten Disziplin finden und ihn zugleich laufend erweitern und neu definieren.

5) Der Bezug zu Manipulation und Ethik ist auffällig: Es fällt auf, dass Public Relations oft in deutlich negativem Zusammenhang thematisiert werden: Gern wird von Schönfärberei, von unbewusster und bewusster Täuschung (Manipulation) der Öffentlichkeit (vgl. so Habermas 1995: 446), von inszenierten Pseudo-Ereignissen, von Schein statt Sein gesprochen, und oft gibt es spektakuläre Ereignisse, die diese negative Sicht öffentlich verstärken (vgl. hierzu etwa Becker-Sonnenschein/Schwarzmeier 2002). Das bezieht sich sowohl auf die ethische Bedenklichkeit der verwendeten Instrumente als auch auf die Personen, deren Beruf mit ,Public Relations' nur sehr vage umschrieben ist. Möglicherweise erklärt dies auch die besondere Betonung ethischer Standards oder deren Codes oder die auf Öffentlichkeitswirksamkeit zielende Tätigkeit von Gremien, wie etwa dem Deutschen Rat für Public Relations ${ }^{6}$ (DRPR) (vgl. Avenarius 1995: 7ff.).

\section{Probleme mit Definitionen von PR}

Jede Wissenschaft basiert nach Leibniz (vgl. Maser 1971: 18ff.) auf den Elementen Begriff, Satz und Regel, wobei hier der Begriff und seine Definition an erster Stelle ste-

4 Nur als Beispiel: Während Oeckl (1964: 69) noch feststellt, dass Lobbying wenig mit PR zu tun hat, weil „die Schaffung von Vertrauen in diesem Geschäft nicht vorgesehen ist“, gehört es seit etwa 1990 definitiv zu den Instrumenten der PR (vgl. Avenarius 1995: 305ff.)

5 Nur zum Vergleich: Der Beruf des Journalismus hatte ein halbes Jahrtausend Zeit, zu wachsen und sich in der Ära der Printmedien zu konsolidieren. Von Public Relations kann man, von Ausnahmen abgesehen (vgl. Kunczik 2002: 108f.) erst zu Anfang des 20. Jahrhunderts sprechen - ausgerechnet in dem Jahrhundert, in dem sich fast zeitgleich alle elektronischen Massenmedien entwickeln sollten.

6 Dazu Bentele (1996: 144): „Neben der Tendenz, das Thema PR-Ethik wichtiger zu nehmen, existiert allerdings auch eine Tendenz, das Thema PR-Ethik bewusst als Mittel zur ImageVerbesserung ,vor den Kulissen’ einzusetzen, wobei ,hinter den Kulissen’ eine spezielle PREthik für unrealisierbar oder unrealistisch gehalten wird.“ 
hen. ${ }^{7}$ Definitionen von Begriffen sind zugleich die Basis aller Theorie. „Begriffsbildung und Theoriebildung [sind] in der Wissenschaft so eng miteinander verflochten [...], dass sie im Grunde genommen zwei unterschiedliche Aspekte der gleichen Vorgehensweise ausmachen" (Hempel 1974: 13). Solange über das Erkenntnisobjekt einer Wissenschaft substanziell gestritten wird, wird es mindestens so viele Definitionen wie Erkenntnisobjekte geben. Insofern ist die Zahl von Definitionen zugleich ein verlässlicher Indikator für den Reifegrad einer Wissenschaft selbst: „Begriffe können Indikatoren für die Realentwicklung von Phänomenen sein“ (Bentele 1997a: 141).

Die exakten Naturwissenschaften, in diesem Kontext stets als Vorbild bewundert, gelten deshalb als exakt, weil ihre Dimensionen entweder logisch (so die reine Mathematik) oder aber als beliebig präzise empirische Erfahrungswerte (etwa: die Berechnung der Zahl $\varpi)$ bestimmt werden können. Folglich gibt es hochverbindlich vereinbarte Dimensionen, wie etwa das Zentimeter-Gramm-Sekunden-System, und andere, an die weitere Dimensionen nahtlos anknüpfen können. Da lebt es sich, wissenschaftssoziologisch gesehen, kommod. Hier sind die Sozialwissenschaften stark benachteiligt, denn der Grad ihrer formalen Exaktheit ist weit geringer und sie besitzen zugleich einen erheblich komplexeren Erkenntnisgegenstand, der sich bei genauerem Hinsehen nicht nur gegen die sichere Einfriedung im Rahmen einer zweiwertigen Logik sträubt (vgl. dazu Kauffman 1987; Watzlawick et al. ${ }^{2}$ 1971: 32f.), sondern für fast alle seine Variablen nur eine ordinale Skalierung erlaubt. Da lässt die Aufnahme in den Kanon der angesehenen Wissenschaften auf sich warten. Nur als Fingerzeig: Allport (1967) stieß bei der Analyse des Einstellungsbegriffs - damals wie heute ein Schlüsselbegriff der Sozialwissenschaften - auf über 100 Versuche, diesen Begriff zu definieren. Analog lassen sich für den Begriff der Kommunikation mehr als 160 Definitionen ausmachen (vgl. Merten 1977) und für Public Relations zählt Harlow (1976) sogar 472 Definitionen. Das verweist nicht nur auf den noch immer ungesicherten Status einer PR-Wissenschaft, sondern unweigerlich auch auf die Frage, was der Erkenntnisgegenstand von Public Relations eigentlich ist oder - in funktionalistischer Fragestellung - ob die von PR zu erfüllenden Funktionen in genügender Abstraktionslage formuliert werden können.

Die auffällige Heterogenität vorliegender Definitionen von Public Relations ist nicht nur dem noch ungesicherten Erkenntnisgegenstand „Public Relations“ geschuldet, sondern auch den unterschiedlichen Funktionen, die mit der Definition verbunden werden. Definitionsversuche speisen sich (so Rühl 1992: 36; vgl. aber auch Fröhlich 2005: 97ff.) aus drei Quellen (vgl. Abb.1): Zum einen aus den Alltagstheorien („common sense theories“) der PR, als Bemühen festzustellen, „was Public Relations eigentlich ist“. Die zweite Quelle stellt das Arsenal von Theorien angewandter PR („working theories“) dar, wo PR aus der jeweiligen Perspektive des praktischen Arbeitsalltags beschrieben wird - sozusagen als Klärung und Rechtfertigung für das eigene Tun. Die dritte, bislang nur schwach sprudelnde Quelle, stellt das stark heterogene Arsenal (kommunikations-) wissenschaftlicher Theorien dar.

In einem weiteren Schritt (vgl. Abb. 1) kann man die vorfindlichen Definitionen tentativ weiter differenzieren in:

1) Alltagsdefinitionen, die einen singulären relevanten Aspekt betonen: „PR is the art of opening men's minds“ (USA, hier zitiert bei Oeckl 1964: 32) oder „to influence public opinion“ (zitiert bei Cutlip et al. 1994: 2).

7 Natürlich gilt aber, dass man Regeln braucht, wie Definitionen anzufertigen sind, und Sätze, was Regeln besagen sollen. Aber das ist ein Problem, das im Rahmen des hermeneutischen Zirkels gelöst wird. 


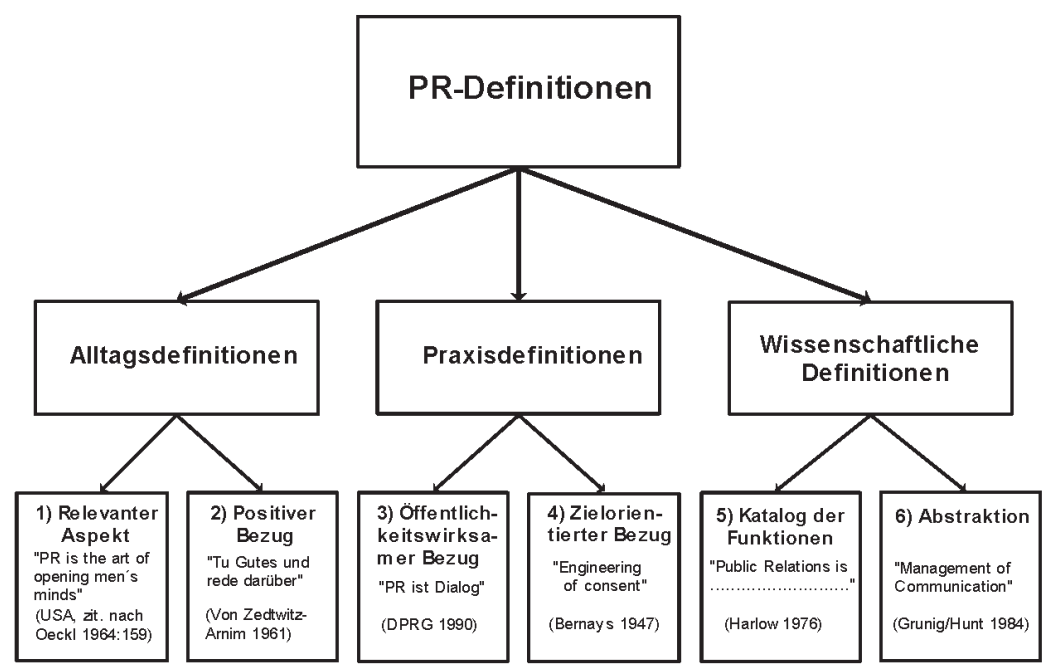

2) Alltagsdefinitionen, die den positiven Bezug von Public Relations betonen: Die bekannteste Definition dieser Art ist die von Zedwitz-Arnim (1961): „Tu Gutes und rede darüber". Diese Definition erschöpft sich zudem in der Formulierung einer Norm für das Handeln von PR-Fachleuten, sie leistet keinerlei Beschreibung.

3) Praxis- oder Praktikerdefinitionen, die für die Öffentlichkeit formuliert werden: Das sind vorzugsweise Definitionen der PR-Verbände, die aus begreiflichem Interesse mit ihren PR-Bemühungen schon bei der Definition von PR ansetzen, also bereits PR für PR machen (vgl. so bereits Scharf 1971: 167). Die Deutsche Public Relations Gesellschaft DPRG (1990) definiert PR in Anlehnung an die Modelle von Grunig/Hunt (1984) zunächst unerschrocken als „Dialog“. Dieser Begriff ist deutlich positiv besetzt - aber natürlich kann PR niemals Dialog sein. Im Jahr 2005 wird der Dialog ersetzt durch die aus der gleichen(!) Publikation von Grunig/Hunt (1984: 6) stammende Definition von PR als „Management (die Regelung) von Kommunikation“, die mittlerweile ebenfalls positive Assoziationen entfaltet (vgl. DPRG 2005: 8). Aus weiteren solcher Beispiele kann man schließen, dass Standesorganisationen zum einen der aktuellen Entwicklung von Definitionen mit einer gewissen Verzögerung stets folgen und dabei gern einer positiv getönten Variante den Vorzug geben.

4) Praxis- oder Praktikerdefinitionen, die das Gesamtziel von PR-Bemübungen artikulieren: Hier wird PR unter einer Gesamtperspektive definiert, beispielsweise als „Engineering of consent" (Bernays 1947) oder als ,inducing the public to have understanding for and good will“ (zitiert bei Cutlip et al. 1994: 2).

5) Wissenschaftliche Definitionen, die in einem ersten Anlauf einen Katalog von Funktionen definieren, die Public Relations erfüllen resp. erfüllen sollen: Funktionen gelten zu Recht als theoretische Begriffe, insbesondere in den Sozialwissenschaften. Man versteht darunter im einfachen Verständnis eine Aufgabe (Soll) oder Leistung (Ist), die ein System bzw. ein Mechanismus erbringen soll oder erbringt. Aber auch Zweck, Ziel, Konsequenzen, Motive werden synonym zum Begriff der Funktion gebraucht (vgl. Merton 1967: 123). In der Technik und in den Sozialwissenschaften ist dagegen 
mit Funktion die Erbringung einer Leistung in Bezug auf eine bestimmte Dimension gemeint: So hat etwa eine Schraube die Funktion der Verbindung von mehreren Teilen und so haben Gesetze die Funktion, ein geordnetes Leben der Mitglieder einer Gesellschaft zu ermöglichen.

Sucht man also nach Funktionen von PR, so wird man schnell fündig, denn als Funktionen von Public Relations für eine Organisation oder eine Person werden vor allem 1) die Steigerung des Bekanntheitsgrades, 2) die Verbesserung von Images, 3) die Erzeugung von Vertrauen, 4) die Legitimierung unternehmerischen Handelns, 5) die Akzeptanz in der Öffentlichkeit und 6) die Vergrößerung von Handlungsspielräumen genannt (vgl. statt anderer Avenarius 1995: 20f.; Röttger 2000: 79ff.; Wienand 2003: 152ff.; DPRG 2005: 11).

Harlow (1976: 36) definiert in diesem Sinne wie folgt: „Public relations is a distinctive management function which helps establish and maintain mutual lines of communication, understanding, acceptance and cooperation between an organization and its publics; involves the management of problems or issues; helps management to keep informed on and responsive to public opinion; defines and emphasizes the responsibility of management to serve the public interest; helps management keep abreast of and effectively utilize change, serving as an early warning system to help anticipate trends; and uses research and sound and ethical communication techniques as its principal tools."

Der damit präsentierte Katalog von $17 \mathrm{zu}$ erfüllenden Funktionen (die allerdings nur aufgezählt, aber nicht begründet oder zueinander in Beziehung gesetzt werden) markiert den typischen Katalog der Funktionen, der sich im eher akzessorischen Aufzählen von zu erbringenden Leistungen erschöpft. Zum anderen aber wird PR hier als „spezifische Managementfunktion“ bezeichnet, die diese Forderungen erfüllen soll. Doch ein Prinzip, wie dies genau vonstatten gehen soll und ob das für alle zu erbringenden Leistungen gelten kann, wird nicht formuliert. Der Bezug auf Kommunikation ist eher vordergründig, die Schaffung und Sicherstellung von Kommunikation ist nur eine unter vielen anderen Funktionen.

Das Problem ist jedoch, dass Funktionen stets nur die Lösung einer Aufgabe unter einer jeweils gewählten Perspektive beschreiben und damit Alternativen zulassen. Die Aufzäblung von Funktionen kann daher niemals erschöpfend sein (denn es gibt so viele Funktionen wie es Perspektiven gibt, also: unendlich viele!), so dass eine Definition von PR durch Aufzählung von Funktionen niemals abgeschlossen sein kann.

6) Wissenschaftliche Definition als Abstraktion: Hierunter sind Definitionen zu rechnen, die statt einer auf Vollständigkeit zielenden Aufzählung von Funktionen eine Funktion auf abstrakterer Ebene nennen. Exemplarisch dafür stehen Grunig und Hunt (1984: 6), die Public Relations definieren als „management of communication between an organization and its publics" und somit präzise sagen, was zu managen ist: Kommunikation. Auch die Definition von PR durch Merten/Westerbarkey (1994: 210) gehört hierher, denn diese Autoren sehen eine übergeordnete Funktion der PR, die sie als „Prozess intentionaler und kontingenter Konstruktion wünschenswerter Wirklichkeiten" definieren.

\section{Implikationen für eine Definition von PR}

Im Versuch, den Begriff Public Relations zu definieren, wurden hier bislang a) Schwierigkeiten bei der Definition von PR und b) Schwierigkeiten mit Definitionen von PR vorgestellt in der Hoffnung, dabei grundlegende oder für PR ,typische' Bedingungen, Eigenheiten, Prinzipien oder Strukturen zu finden, unter denen PR gefasst werden kön- 
nen. Solche Versuche verlangen Kreativität, und die dabei erzielten Lösungen erscheinen daher oft willkürlich oder gar unverständlich, doch ihnen liegt implizit eine gewisse Methodik zugrunde ${ }^{8}$. Bei diesem hier angestellten Versuch schälen sich sechs Befunde heraus, die im Folgenden festgehalten werden sollen:

In sehr unterschiedlichem Gewand (etwa: Bewusstsein [mind], Pseudo-Event, Image, Öffentlichkeit, Inszenierung) tauchen häufig Begriffe auf und werden für wichtig erklärt, die stark fiktionale Qualitäten besitzen.

Die Wirkung der (eingesetzten Kommunikationsprozesse für) PR soll langfristig vor allem in der Schaffung von mentalen Qualitäten, nämlich der Erzeugung von Überzeugungen, Glaubwürdigkeit und Vertrauen bei den jeweiligen Zielgruppen bestehen.

Public Relations basieren auf vorsätzlich und gezielt gesteuerten Kommunikationsprozessen (management of communication).

Public Relations sind nicht einfach singuläre Kommunikationsprozesse, sondern ein komplexes Arrangement von kontinuierlich erzeugten, miteinander verschränkten und zueinander passenden Kommunikationsprozessen mit Optionen für Anschlusskommunikation.

Public Relations soll für bestimmte Objekte bei vorab als relevant definierten Öffentlichkeiten (Zielgruppen) tendenziell positivere Wahrnehmungen bzw. positivere Wirklichkeitskonstruktionen generieren.

Wie keine andere Kommunikationstätigkeit werden PR laufend negativ assoziiert mit Begriffen wie Täuschung, Manipulation, Schönfärberei, also mit Begriffen, die implizit feblende Wabrbeit oder feblende Wabrhaftigkeit bezeichnen.

\subsection{Fiktionen statt Fakten}

Setzt man zunächst am Begriff der Fiktion an, so ist ein Bezug zur Mediengesellschaft ${ }^{9}$ schnell hergestellt: Parallel zu ihrer Entstehung weitet sich, wie bekannt, der Bedarf für PR systematisch aus, weil mediale Inhalte grundsätzlich, fiktional' sind und die dadurch transportierbaren Images von Personen, Unternehmen, Ereignissen oder Ideen als solche schnell und kostengünstig aufgebaut und auch verändert werden können durch eine neue Profession, die die Konstruktion von Wirklichkeit als ernst zu nehmenden Beruf

8 Sie verlangt vor allem a) eine möglichst gute Übersicht bzw. Kenntnis des zu bearbeitenden Erkenntnisgegenstands, b) die Fähigkeit, in vorliegenden Variationen von Elementen, Relationen oder Strukturen zugrunde liegende Gemeinsamkeiten zu erkennen, c) spontane Annahmen aufzustellen, aber d) auch deren Prüfung zu riskieren und e) ggf. auf höherem bzw. abstrakterem Level ein übergeordnetes Ganzes zu erkennen. Das bedeutet stichprobentheoretisch u. a. auch die Fähigkeit, eine auf Repräsentativität zielende Auswahl der vorfindlichen Erscheinungen zu leisten.

9 Von „Mediengesellschaft“ kann man sprechen, wenn zwei Bedingungen erfüllt sind: 1) das Auftreten von Metamedien (etwa: Programmzeitschriften, Suchmaschinen etc.) zur Raffung von Medieninhalten im großen Stil und 2) das Anwachsen von Fiktionen, die massive faktische Wirkungen entfalten und nun gleichberechtigt neben Fakten stehen können. Für den Beginn der Epoche der Mediengesellschaft in der Bundesrepublik Deutschland kann man hilfsweise das Jahr 1984 ansetzen - das Jahr also, in dem das Duale Rundfunksystem implementiert wird (vgl. dazu Merten 2005) 
betreibt: Public Relations. Ausschlaggebend dabei ist, dass Sachverhalte aller Art allein durch ihre mediale Behandlung eine massive Aufwertung an Relevanz erfahren, die publikumsseitig als Erwartung für Unerwartetes (Aktualität) einerseits und zugleich als gesamtgesellschaftliches Relevanzmodell gelernt wird: Was nicht in den Medien ist, ist nicht relevant.

Zugleich wächst damit der Druck, in den Medien vertreten zu sein, denn nur wer dort sichtbar ist und souverän auftritt, gilt auch als existent, als wahrnehmbar, als souverän. Die Verhältnisse drehen sich geradezu um: Nicht der ist gut aufgestellt, der wirklich gut aufgestellt ist, sondern der, der in den Medien wirklich gut aufgestellt erscheint.

\section{Tabelle 1: Typen von Fiktion als generalisierte Stellvertreter in der Mediengesellschaft}

Frühere Gesellschaften

Mediengesellschaft

\section{FAKT $\rightarrow$ FIKTION}

Objektive Wirklichkeit („Realität“)

Ereignis

Sachverhalt

Person

Zu lösendes Problem

Wahrheit

Autoptische Beobachtung

\author{
Medialer Wirklichkeitsentwurf \\ Event, Bericht über Ereignis (Text) \\ Inszenierung \\ Image \\ $\mathrm{Zu}$ diskutierendes Thema \\ Öffentliche Meinung \\ Beobachtung der Beobachtung
}

Diese Struktur hat dazu geführt, dass die Darstellung von Personen und Sachverhalten in den Medien längst als Stellvertreter für die eigentliche Person bzw. den eigentlichen Sachverhalt gesehen werden kann (vgl. Tab. 1): Bei Personen nennen wir den medialen Stellvertreter Image, bei einem Sachverhalt sprechen wir auch von dessen Inszenierung. Wir ersetzen Wahrheiten durch das Votum der öffentlichen Meinung und unsere eigenen Beobachtungen durch die Beobachtungen der Medien, die wir dann beobachten. Parallel dazu entwickeln sich weitere Stellvertreter: Wahrheit kann nun durch die Unterstellung von Wahrheit, Glauben durch die Unterstellung von Glauben ersetzt werden etc. Man erkennt, dass die Kommunikation in der Mediengesellschaft unter den vielen Innovationen auch die Konstruktion von Stellvertretern und die Möglichkeit, wirksam zu unterstellen, hervorbringt.

Zwar setzt die Nutzung von Fiktionen im großen Stil für PR erst in der Mediengesellschaft ein, doch ihre Ursprünge liegen tausende Jahre zurück, nämlich beim ersten kommunikativen Modus, der Propaganda (publicity). Zu der Zeit hat der Prophet Mose, zweifelsfrei der Ahnherr des Kommunikationsmanagements überhaupt, sein ,Unternehmen', nämlich den Zug ins ,gelobte Land', vierzig Jahre geführt und dabei grundlegende Prinzipien des Kommunikationsmanagements formuliert und angewandt, um sein Volk von seiner Vision einer invisiblen Gottheit zu überzeugen. Die von ihm entwickelten Prinzipien, Kommunikation wirkungsvoll (überzeugend) zu gestalten, sind 
erstaunlich genau beschrieben und befestigen das Propagandamodell der $\mathrm{PR}^{10}$, das über Jahrtausende vorherrschend sein wird (vgl. als ersten Hinweis Oeckl 1964: 79, im Detail sodann Fischer-Appelt 2005 sowie Merten 2008). Zentral ist dabei das von Mose erstmals genutzte Prinzip, mit Fiktionen Fakten zu erzeugen und die darauf basierende Anleitung zum Kommunikationsmanagement ${ }^{11}$ für unwiderstebliche Kommunikation - ein Gedanke, der viel später im Gewand des Konstruktivismus eine spektakuläre Wiedergeburt erfährt.

Das Aufkommen der Medien führt später zwar zu drei weiteren Modi der Kommunikation (vgl. Grunig/Hunt 1984: 21ff.), doch bis heute wird der Modus der Propaganda in der PR häufiger verwendet als der Dialog-Modus (siehe Avenarius 1995: 85). Und gerade das Aufkommen der Medien begünstigt die Verwendung von Fiktionen in der PR und die damit erzielbaren Wirkungen, es begünstigt ebenfalls die Erzeugung von Glaubwürdigkeit, Überzeugungen und Vertrauen.

\subsection{Alle PR basiert auf Kommunikation}

Der Bezug aller Public Relations auf Kommunikation wird hier nicht von ungefähr betont, denn die wesentlichen Kernkompetenzen von PR, vor allem Kontakt, Redaktion, Konzeption und Management (vgl. Wienand 2003: 152ff.; DPRG 2005: 11), sind sämtlich Kompetenzen, die eine generelle kommunikative Kompetenz einfordern und zugleich wesentliche, der PR abzufordernde Funktionen (Leistungen) markieren ${ }^{12}$. Genau an diesem Bezug zur Kommunikation lässt sich eine weitere Argumentationslinie entfalten.

Zum einen: Wenn man die Spezifik kommunikativer Leistungen ermitteln will, ist es aufschlussreich, sie mit nichtkommunikativen Leistungen $\mathrm{zu}$ vergleichen ${ }^{13}$. Vergleicht man also „normale“, laufend zu erbringende PR-Leistungen mit „normalen“ NichtPR-Leistungen (etwa: mit der Herstellung von Industrieprodukten), so besteht ein entscheidender Unterschied offenbar darin, dass bei der Herstellung von Produkten - vom Handwerk bis zur Hochtechnologie - in der Regel alles getan wird, um völlig gleiche Beschaffenheit sicherzustellen: Man setzt strengstens auf Konfektion. Dagegen

10 Bentele (1997: 25) erkennt abweichend von Grunig/Hunt (1984: 21ff.) Propaganda wegen ihrer Einweginformation und ihrer mangelnden Wahrheit nicht als Modus von Public Relations an. Wie im Folgenden zu zeigen sein wird, ist aber gerade der Verzicht auf unbedingte Wahrheit und Wahrhaftigkeit eine notwendige Voraussetzung für Public Relations, insbesondere wenn sie erfolgreich sein will.

11 Mose zeigt, analytisch gesprochen, dass der Glauben dann trägt, wenn man an den eigenen Glauben glaubt, dass Überzeugungen dann wirksam sind, wenn man von deren Wirken überzeugt ist, dass Vertrauen auf Gott stärkt, wenn man seinem Vertrauen traut etc. Vgl. auch Fischer-Appelt (2005: 146).

12 Damit ist allerdings nicht der in der PR-Praxis zu beobachtende Trend gemeint, den Begriff „Public Relations“ durch die positiver besetzten Begriffe „Kommunikation“ oder „Dialog“ zu ersetzen.

13 Wissenschaftstheoretisch ist der Vergleich nicht nur die einfachste Form der Differenzbildung, sondern damit eng verknüpft auch der einfachste Typ von Messung, der in jedem Fall heuristisches Potenzial besitzt. Die hier durch den Vergleich vorgenommene Differenzbildung eröffnet eine unerwartete Perspektive: Differenzbildung als Prinzip. Dieses Prinzip generiert unvermeidlich weitere Differenzbildung a) in verschiedenen Dimensionen und b) als Folge dieser Differenzbildung - womit das genetische Prinzip von Bateson (1981: 25f.) auch hier eine interessante Bestätigung findet. 
sind Kommunikationsmanager genau umgekehrt darauf geeicht, jeweils Unikate zu erzeugen. ${ }^{14}$ Das ergibt sich direkt aus der Tatsache, dass Leistungen von PR stets in der Herstellung bzw. Aufrechterhaltung von Kommunikation mit (mindestens) einem Kommunikanten liegen, dessen Beitrag nicht kalkulierbar und schon gar nicht ignorierbar ist und daher selbst bei identischen Rahmenbedingungen stets zu einem differenten Ergebnis führt. Von daher wäre eine Standardisierung nicht nur kontraproduktiv, sondern sie wäre der sichere Tod aller PR. Abstrakter gesprochen: Differenzbildung in der Kommunikation stellt offenbar ein originäres Prinzip von Public Relations dar.

Eine weitere Argumentationslinie ergibt sich, wenn man erneut von der Kommunikation ausgeht und fragt, was deren „Management“ bedeutet. Ausgangspunkt dafür ist die Definition von Grunig/Hunt (1984), die zwar den Managementbegriff einführt, aber diesen und die damit zu bewirkende Leistung nicht expliziert. Holt man dieses Versäumnis nach, dann stellt man überrascht fest, dass Manager in allen beruflichen Branchen definitiv nur eine einzige dominante Tätigkeit ausüben: Kommunikation. Neun von zehn der nach Mintzberg (21980: 37) von Managern auszufüllenden Rollen entpuppen sich schon bei erster Hinsicht als lupenreine Kommunikator-Rollen, und bereits 1975 verwendeten Manager im Durchschnitt 80 \% ihrer Zeit auf Kommunikation - ein Wert, der bis zum Jahr 2000 auf über $90 \%$ ansteigt (vgl. Mintzberg ${ }^{2}$ 1980: 42; Oelert 2003: 2).

Management von Kommunikation ist also längst ein Management von Kommunikation durch Kommunikation. Das heißt nichts anderes, als dass PR-Fachleute (Kommunikationsmanager) Metakommunikatoren sind: Sie setzen Kommunikation (als Mittel) ein, um Kommunikation (als Ziel) ins Werk zu setzen. Dass dies ausgerechnet bei Kommunikation eintritt, ist freilich kein Zufall, denn Kommunikation kann sich nicht nur über alle denkbaren Themen hermachen, sondern auch - autologisch - über sich selbst. Gerade darin liegt ja ihr besonderes Potenzial.

Dieser Befund liefert sogleich einen weiteren Fingerzeig, denn die reflexiv erzeugte Zweigliedrigkeit des Prozesses (vgl. Luhmann 1970: 92ff.) beschafft Zeit und installiert Freiheitsgrade, so oder auch anders, also: kontingent zu kommunizieren. Sie macht mithin gerade die Management-Leistung aus, die, aus ganz anderer Perspektive, von Mintzberg (21980: 37ff.) sorgfältig beschrieben wurde.

\subsection{Abgrenzungen zu verwandten Berufsfeldern: Die Elastizität der Wabrheit}

Die letzten beiden der o. a. formulierten sechs Befunde sind heikel, denn sie signalisieren ein offenbar strukturell negatives Image der PR wegen ihres durchgehenden Bestrebens, Sachverhalte stets in positiver Tönung („Schönfärberei“), also letztlich unter Dehnung von Wahrheit und Wahrhaftigkeit, darzustellen. Zwar berichten Journalisten ebenfalls nicht ,neutral', sondern bevorzugt negativ, doch dies basiert auf einer an Relevanz von Sachverhalten orientierten Selektionsentscheidung, nicht auf einer gezielten Täuschung (Manipulation) über zugrunde liegende Sachverhalte selbst. Genau diese Manipulation aber wird dem Wirken von Public Relations - mehr oder minder explizit und durchgängig - angelastet, und dagegen wehren sich Public Relations gelegentlich, wenn sie

14 Auch Journalisten erzeugen laufend Unikate. Aber diese werden massenhaft verbreitet und richten sich an ein Gesamtpublikum, dessen persönliche Kenntnis nicht gegeben ist. Künstler verfertigen ebenfalls Unikate, aber sie kennen den späteren Käufer vorher nur im Ausnahmefall. 
- zumindest öffentlich oder standesbezogen - den Vorwurf mangelnder Wahrhaftigkeit für nicht zutreffend erklären und diesen somit erst recht befestigen.

Die letzten beiden Befunde stimulieren jedoch eine ganz andere Deutung, wenn man einen Blick auf die benachbarten Berufsfelder des Journalismus und der Webung riskiert: Üblicherweise gilt als gesichert, dass der Journalismus auf Objektivität und Wahrheit seiner Inhalte abonniert ist und dies offen für sich reklamiert. Nicht umsonst lautet das Credo des anglikanischen Journalismus „comments are free but facts are sacred“. Auf der anderen Seite steht die Werbung, die keinerlei Wahrheitsansprüche für sich reklamiert, sondern ganz im Gegenteil deren Absenz offen zugibt. Zwischen beiden bewegen sich Public Relations: Sie fordern für bestimmte Situationen, z. B. in Fällen von Krisen-PR, die Beachtung uneingeschränkter Wahrhaftigkeit und sie fordern in anderen Konstellationen, z. B. bei Skandalen aller Art, notfalls auch die perfekte Unwahrheit (vgl. Abb. 2).

\section{Abbildung 2: Schnittmengenmodell von Journalismus, Public Relations und Werbung}
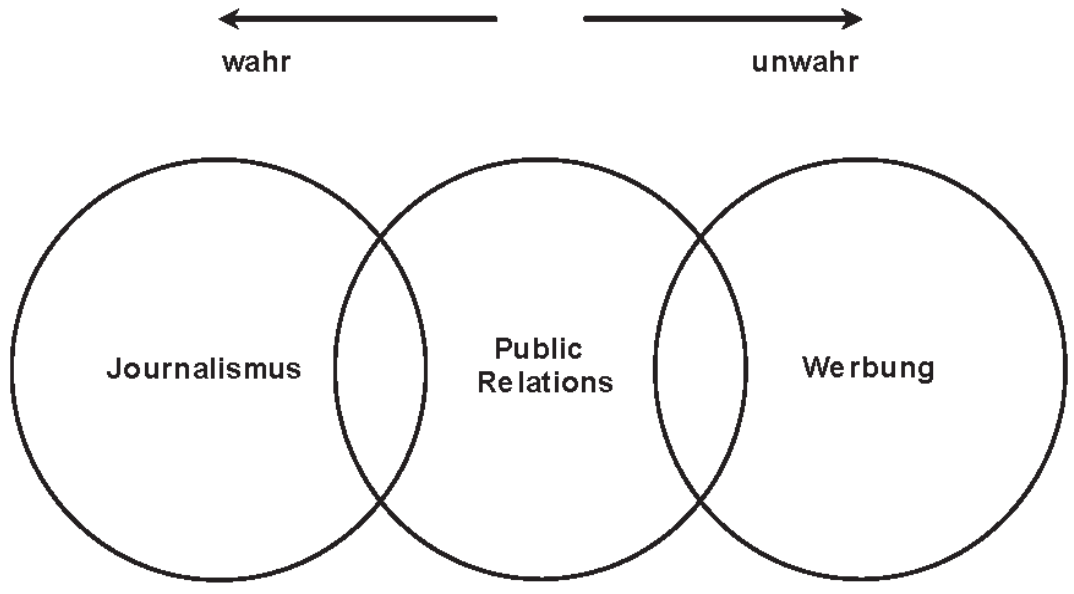

Denn Public Relations sind darauf angewiesen, Wirklichkeiten (Sachverhalte) fallbezogen so oder auch anders, also: kontingent darzustellen. Ihre Aufgabe liegt nicht in der strikt wahrheitsbezogenen Darstellung von Sachverhalten, sondern in deren situational bedingter Anpassung. PR-Manager müssen dabei diese Elastizitäten bis zu deren Grenzen nutzen, um die geplante Wirkung ihrer Kommunikation bei den jeweiligen Zielgruppen zu erreichen, ohne ihre Glaubwürdigkeit zu verlieren. Fachleute für PR sind, so gesehen, nichts anderes als professionelle Konstrukteure fiktionaler Wirklichkeiten, sind Experten darin, die Semantik einer Sache zu der Sache selbst in kontingenter Distanz zu verhandeln (vgl. Merten 2007: 27).

Das ist ein schwieriges und sehr verantwortungsvolles Geschäft und bedeutet keinesfalls, dass PR-Fachleute stets lügen müssen. Aber sie dürfen auch nicht stets die Wahrheit sagen. Diese Feststellung mag befremdlich erscheinen, aber ein einziger Blick etwa auf die Diplomatie zeigt, dass dieses Problem auch in anderen Teilsystemen bekannt ist und seine Lösung gesellschaftlich wenn nicht gebilligt, so doch verstanden wird.

PR lassen sich weder auf Wahrheit noch auf Unwahrheit festlegen, weil sie auf differente Situationen different reagieren und daher auch mit Wahrheiten elastisch umge- 
hen müssen. Die Hintanhaltung von Information, in der PR geradezu als Maßstab für Professionalität gehandelt, ist sowohl in der Werbung als auch im Journalismus sinnlos oder sogar kontraproduktiv und daher dort unbekannt. Während PR sich an viele Zielgruppen $^{15}$ wenden, wendet sich Werbung nur an eine einzige Zielgruppe: den Konsumenten. Während der Begriff der Wahrheit für Werbe-Aussagen irrelevant ist, ist er für PR-Aussagen existenziell (vgl. Tab. 2).

Und während der Journalismus zu Recht seinen Wahrheitsanspruch fixiert (wer würde sonst z. B. noch Nachrichten lesen, hören oder sehen wollen?) und die Werbung umgekehrt den fehlenden Wahrheitsanspruch ihrer Kommunikation offen zugibt (vgl. dazu vor allem Schmidt 1995), oszillieren Public Relations zwischen diesen Polen, indem sie eine diesbezügliche Festlegung auf Wahrheit oder Nicht-Wahrheit kategorisch unterlaufen müssen, um situationsabhängig flexibel reagieren zu können. Oder anders: Sie lassen sich weder auf Wahrheit noch auf Unwahrheit festlegen, weil sie auf differente Situationen different reagieren und daher auch mit Wahrheiten elastisch umgehen müssen (vgl. Tab. 2). Hegelianisch könnte man auch sagen, dass PR sich laufend zwischen den Polen nicht nicht wahr und nicht nicht unwahr bewegen müssen.

\section{Tabelle 2: Journalismus, $P R$ und Werbung in differenztheoretischer Perspektive}

$\begin{array}{ll}\begin{array}{l}\text { Eigenschaft } \\ \text { Präferenz }\end{array} & \begin{array}{l}\text { Journalismus } \\ \text { negativ }\end{array} \\ \begin{array}{l}\text { Frequenz der Aus- } \\ \text { sagen }\end{array} & \text { einmal } \\ \begin{array}{l}\text { Integration/Differenz } \\ \text { Differenzierung }\end{array} & \text { integrativ } \\ \begin{array}{l}\text { zeitlich } \\ \text { Differenzierung }\end{array} & - \\ \text { sachlich } & - \\ \text { Differenzierung sozial } & \text { alle } \\ \text { Wahrheitsanspruch } & \text { definitiv JA } \\ \text { (objektiv) } & \end{array}$

Public Relations
positiv
kontingent
differenzierend
vor/nacheilend/nie
so oder anders
kontingent nach Zielgrup-
pen
elastisch

Werbung
positiv
immer
integrativ
-
-
eine Zielgruppe
(undifferenziert)
definitiv NEIN

Schließlich zeigt auch die systemtheoretische Analyse von Kommunikation, dass alle Kommunikationsprozesse primär bestrebt sind, sich im Sinne der „latent pattern maintenance“ (vgl. Parsons 1959: 7) selbst zu erhalten und dass sie daher gegen Wahrheiten strikt indifferent sein müssen. Von daher betreiben alle Public Relations in der je situational gebotenen Differenzierung von Wahrheit genau das, was Kommunikation am Leben erhält - und auch das ist keine geringe Leistung.

\section{Resümee}

Die hier zusammengetragenen Befunde legen es nahe, Public Relations unter erheblich abstrakterer Perspektive zu formulieren. Zieht man das hier skizzierte Potenzial differenter Darstellung in den Begriff des Kommunikationsmanagements hinein, so bietet

15 Das Konzept der Zielgruppe wurde vom Marketing konsequent auch für den Kommunikationsmix angewendet. Vgl. dazu bereits Lentz (1978: 16) sowie später ausführlich Meffert (2000: 682ff.). 
sich eine differenztheoretische ${ }^{16}$ Perspektive an: Public Relations sind ein Typ von Differenzmanagement. Genauer: Public Relations sind darauf geeicht, die Wahrnehmung der Öffentlichkeit in ihrem Sinne zu manipulieren, und dies gelingt ihnen durch eine entsprechend differente Kommunikation. $\mathrm{Zu}$ deren Strukturierung bietet sich das zeitlich-sachlich-soziale Dimensionsschema an, das von Luhmann (1970: 120ff.) als systemische Grundarchitektur zur Generalisierung von Verhaltenserwartungen vorgeschlagen wurde.

Ein zeitliches Differenzmanagement der PR lässt sich unschwer orten, weil gerade in der PR mit der Variablen ,Zeit' hochsensibel umgegangen wird: So besagt eine der Grundweisheiten praktischer PR, dass es eine richtige Zeit gibt, Dinge zu sagen und zu tun, aber eben auch eine falsche Zeit. Pressemitteilungen über irgendein Ereignis lassen sich bei Bedarf beschleunigen, gar vor dem eigentlichen Ereignis platzieren (gerade deswegen benötigt die PR ja fiktionale bzw. Pseudo-Ereignisse), aber bei Bedarf auch verzögern oder hintanhalten, so dass im Idealfall eine Berichterstattung sogar verhindert werden kann („nie“).

Das Differenzmanagement von Public Relations in sozialer Dimension zeigt sich als Differenzierung der Kommunikation nach Zielgruppen. Es ist uns so vertraut, dass die eigentliche, jeweils neu zu leistende Differenzierungsleistung - eben: die laufende Erzeugung von Unikaten - gar nicht mehr bewusst wird.

Das sachliche Differenzmanagement verlangt die Darstellung eines Sachverhalts wiederum in der Formel „So, oder auch anders" und ist bereits bei Mose dem Propheten als „Konstruktion von Wirklichkeit durch Fiktionen“ in Erscheinung getreten. Dieser Typ von Differenzmanagement lässt sich auch als Kontingenz- oder Evidenzmanagement von Fakt und Fiktion begreifen. Genau hier, an dieser Dimension, setzt bei Public Relations die heikle Frage nach dem erlaubten Grad von Täuschung an, der wiederum auf die Kategorie reflexiven Vertrauens verweist.

Oder anders gesagt: Kommunikationsmanager müssen stets in zwei Dimensionen gleichzeitig optimieren: Zum einen sollen sie im Sinn ihrer Bemühungen optimale kommunikative Wirkungen bei der Veränderung der Wahrnehmung der Offentlichkeit oder der relevanten Zielgruppen erzielen und zum anderen sollen sie ihre Glaubwürdigkeit niemals riskieren. Der perfekte Fall, dass man die Öffentlichkeit über Täuschungen zu täuschen versteht, kann, anders als bei der Geheimhaltung von Geheimnissen (vgl. Westerbarkey 1998: 98f.), nicht ex ante garantiert werden.

Dahinter steht die in allen Kulturen zu beobachtende Alltagserfahrung, dass die Öffentlichkeit unter bestimmten Randbedingungen ganz offensichtlich die schiere Wahrheit scheut und - innerhalb eines akzeptablen Konfidenzintervalls - viel besser auf Varianten freundlicher Täuschung anspricht. „Mundus vult decipi“ - diese Weisheit war schon im alten Rom gängig. Dazu Westerbarkey (1998: 99): „Wiederum zeigt sich, dass Geheimhaltung und Täuschung für den Bestand von Beziehungen von zentraler Bedeutung sind, so dass wir manchmal sogar anderen helfen müssen, uns selbst zu täuschen oder zumindest den Schein zu wahren." Dass sich die herkömmliche Ethik der Public

16 Die Differenztheorie gilt als ein Typ von Supertheorie, weil sie in mehr als eine Disziplin hineinreicht. Eines ihrer Grundprinzipien wurde von Bateson (1981: 408) wie folgt formuliert: „Information lässt sich definieren als ein Unterschied, der einen Unterschied macht“. 
Relations mit einer professionell eingesetzten Elastizität von Wahrheit ausgesprochen schwer tut ${ }^{17}$, sagt nichts gegen die Viabilität dieses Prozedere.

Natürlich erfordert ein sinnvolles Differenzmanagement stets die Gleichzeitigkeit zeitlich-sachlich-sozialer Differenzbildung: Es gilt stets, zur richtigen Zeit die richtige Zielgruppe mit dem richtigen Thema anzusprechen und eine viable Kommunikation zu entwickeln. Und es gilt in der PR ebenso als Leistung, flexibel zu reagieren, also das Differenzmanagement sogar selbst noch zum Gegenstand sinnvoller Differenzbildung zu machen - zum Beispiel in der Krisen-PR. Und umgekehrt: Keine Kommunikation kann größeres Unheil anrichten als die, die mit dem falschen Thema zur falschen Zeit an die falsche Zielgruppe gerichtet wird. Wir können daher Public Relations nun wie folgt definieren:

Public Relations sind das Differenzmanagement zwischen Fakt und Fiktion durch Kommunikation über Kommunikation in zeitlicher, sachlicher und sozialer Perspektive. ${ }^{18}$

Die Frage ist, welche Vorteile diese Perspektive eröffnet. Zumindest drei Optionen lassen sich benennen: Zum einen wird damit der (mögliche) Anschluss an eine genügend abstrakte Theorie hergestellt, nämlich die Differenztheorie, die als Weiterentwicklung der Systemtheorie gelten kann (vgl. Luhmann 2003: 76ff.). Public Relations basieren auf einer sinnvollen Differenzbildung in zeitlich-sozialer-sachlicher Hinsicht. Begreift man Kommunikation als System, so ist einsichtig, dass eine PR-Definition, die differenztheoretisch verankert ist, weitere theoretische Anschlussmöglichkeiten offen hält. ${ }^{19}$ Differenzbildung in der PR basiert letztlich auf Kontingenzpotenzial: So, aber auch anders. Mit dieser Kontingenzformel kann ein primärer Zugriff auf Wirklichkeit und eine sekundäre Ausdeutung, die Kommunikationsmanager ${ }^{20}$ stets betreiben müssen, gefasst werden. $^{21}$

Diese Definition hat zweitens den Vorteil, dass sie das Verhältnis von PR zur Werbung und zum Journalismus neu, und das heißt auch: realistischer ordnen kann. Sie kann auf die bislang geläufige Unterscheidung zwischen Überredung und Überzeugung als Abgrenzung von Werbung zu Public Relations einerseits und die Unterscheidung von Information und Überzeugung andererseits verzichten: Kommunikationsmanager

17 Die Ethik der PR, festgeschrieben im Code d'Athènes, folgt einem einfachen, uneingeschränkten und kategorischen Wahrheitsbegriff, der keinerlei Elastizität kennt. Das hat zur Folge, dass die Anwendung auf konkrete Fälle fast unmöglich ist, so dass dieser Code in der Praxis praktisch bedeutungslos geblieben ist (vgl. Avenarius 1998).

18 Prinzipiell verzerrt das Differenzmanagement, bezogen auf den Auftraggeber, Wirklichkeiten in der Regel eher in positive Richtung, so dass man diese Verzerrung in die Definition mit hineinziehen könnte. Hier wird jedoch davon abgesehen, weil die praktische PR damit erwartbar PR für PR erzeugen würde, so dass diese Definition einen falschen Zungenschlag erhielte. Zum anderen ist nicht immer ausreichend klar, in welcher Richtung die positiven Bezüge zu bilden wären.

19 Die Nutzung einer differenztheoretischen Perspektive ist aufwendig, denn sie setzt die Beherrschung einer mehrwertigen Logik voraus. Das wurde spätestens deutlich, als Luhmann (2003: 66) ein soziales System differenztheoretisch definierte als „Differenz zwischen System und Umwelt".

20 Da es in der PR keinen konsentierten Begriff für die ausgeübte eigentliche PR-Rolle gibt (sondern nur Hilfsbegriffe wie PR-Berater, PR-Fachmann, PR-Schaffender), wird hier der Begriff ,Kommunikationsmanager' gewählt.

21 Dabei ist wichtig, dass dieses "So oder auch anders" nicht mit Beliebigkeiten verwechselt wird: Freiheitsgrade sind auch in der PR eher endlich und aussichtsreiche Alternativen rar. 
sagen zu Recht, dass sie bei Bedarf alle Modi der Kommunikation nutzen müssen, um ihr Ziel zu verfolgen. Bereits Aristoteles argumentiert in seiner Rhetorik, dass Überzeugung auch durch Information (Argumente) beschafft werden kann (vgl. Aristoteles 1995: 32f.). Und natürlich „werben“ Kommunikationsmanager aller Orten für ihre Überzeugungen. ${ }^{22}$

Konnte man der o. a. Definition von Grunig/Hunt noch vorwerfen, dass sie auch auf Werbung passt - was die Verfasser sogar selbst einräumen (vgl. Grunig/Hunt 1984: 22), so vermeidet die hier entwickelte differenztheoretische Definition von PR die Anziehung einer Unterscheidung zwischen Überredung und Überzeugung definitiv: Die Hintanhaltung von Information, in der PR geradezu als Maßstab für Professionalität gehandelt, ist in der Werbung sinnlos und daher unbekannt. Während PR sich an viele Zielgruppen wendet, wendet sich Werbung nur an eine einzige Zielgruppe: den Konsumenten. Während der Begriff der Wahrheit für Werbe-Aussagen irrelevant ist, ist er für PR-Aussagen existenziell, weil deren differenzierter Einsatz nun das eigentlich wirksame Kriterium abgibt.

Drittens: Wenn Differenzbildung stets eine weitere Differenzbildung nach sich zieht (vgl. so erstmals Bateson 1981: 25ff.), wenn Differenzbildung sozusagen die autopoetische Laufmasche der Kommunikation abgibt, weil Information die kleinste Einheit der Differenzbildung ${ }^{23}$ darstellt (vgl. so schon Shands 1967), dann sind gerade Kommunikationsmanager gut beraten, vom Differenzmanagement allerbesten Gebrauch zu machen. In der Praxis findet sich dieses Postulat in alltagswissenschaftlichen Forderungen wie beispielsweise „Schweigen ist Silber, Reden ist Gold“ wieder, nicht zufällig steht „storytelling“ (vgl. Posner-Landsch 2007) ganz hoch im Kurs, und Ratschläge wie „Lassen Sie Ihre Kommunikation nie unbeaufsichtigt!“ werden in der PR sehr ernst genommen. Differenzmanagement ist zudem in einem viel weiteren Sinn ein Charakteristikum aller Public Relations. Es ist nicht nur notwendig, um eine effektive, differenzierende Kommunikation zu erzeugen, sondern die dadurch gebildete Differenz ist Ausgangspunkt für weitere mögliche Leistungen der Differenzbildung: Beschaffung von Handlungsspielräumen, Ermöglichung von Kompromissen, Imageveränderung, Change Management etc. Und: Gerade weil Differenzbildung in der PR das Hantieren mit großen Flexibilitäten und hohen Komplexitäten erlaubt, ist ein verlässlicher Bezugspunkt mehr als irgendwo anders notwendig: Die besondere Betonung von Glaubwürdigkeit und Vertrauen in der PR kann, ex iuxtapositione, unter diesem Gesichtspunkt geradezu als empirischer Beleg für diese Zusammenhänge betrachtet werden.

An dieser Stelle müssen notwendig viele Fragen offen bleiben. Ihre Beantwortung setzt - unter anderem - eine stärkere empirische Berufsfeldforschung in der PR, eine methodisch sensiblere PR-Evaluationsforschung und eine längst angemahnte theoretische Konsentierung der Kommunikationswissenschaft voraus. Sie setzt aber vor allem eine anspruchsvollere Ethik der Public Relations voraus, die den Mut besitzt, nicht nur holzschnittartig-dualistisch (ja/nein), sondern different zu befinden: Denn letztlich versteckt sich hinter dem hier gewählten neutralen Begriff „Differenzmanagement“ eine

22 Der Begriff der Überzeugung ist wissenschaftlich nicht festgelegt. Er findet sich zudem weder in aktuellen noch in älteren Lexika (vgl. etwa Brockhaus-Lexikon ${ }^{18} 1993$ oder Meyers Lexikon ${ }^{8} 1908$ ). Bemerkenswert ist auch, dass der englische Begriff „persuasion“ beides - sowohl Überredung als auch Überzeugung - bezeichnet.

23 An diesem Punkt gibt es noch zu viele Optionen nichtkonsentierter Art. So differenziert Weber (2005: 230) die Distinktion nochmals in Differenz und Unterscheidung. 
Technik bedingt geduldeter öffentlicher Täuschung mit autologischer Extension ${ }^{24}$, deren Grenzen bislang noch nicht abgesteckt, geschweige denn verbindlich definiert sind. Wie weit Täuschung gehen darf, hängt offenbar nicht nur vom Grad der Täuschung selbst ab, sondern von der aktuellen Situation, in der die Täuschung stattfindet, sowie von der Relevanz des durch Täuschung zu beschaffenden Nutzens bzw. des dadurch $\mathrm{zu}$ verhindernden Schadens, und dies unter altruistisch/egoistischer Perspektive. Die Schwierigkeiten, hier Grenzen zu definieren, sind einsichtig. Doch das kann allenfalls erklären, aber nicht entschuldigen, dass sich einschlägige Ethik-Kommissionen dieses Themas bislang nie bemächtigt haben.

\section{Literatur}

Aristoteles ( $\left.{ }^{5} 1995\right)$ : Rhetorik (in der Übersetzung von G. Sieveke). München: Fink.

Allport, Gordon W. (1967): Attitudes. In: Carl Murchison (Hrsg.): Handbook of Social Psychology. 2. Band. New York: Russell \& Russell: 798-844.

Avenarius, Horst; Armbrecht, Wolfgang (1992): Ist Public Relations eine Wissenschaft? Opladen: Westdeutscher Verlag.

Avenarius, Horst (1995): Public Relations. Die Grundform der gesellschaftlichen Kommunikation. Darmstadt: Verlag Wiss. Buchgesellschaft.

Avenarius, Horst (1998): Die ethischen Normen der Public Relations. Neuwied/Kriftel: Luchterhand.

Bateson, Gregory (1981): Ökologie des Geistes. Frankfurt: Campus.

Becker-Sonnenschein, Stephan; Schwarzmeier, Manfred (Hrsg.) (2002): Vom schlichten Sein zum schönen Schein? Kommunikationsanforderungen im Spannungsfeld von Public Relations und Politik. Wiesbaden: Westdeutscher Verlag.

Bentele, Günter (1996): Ethik der Public Relations als wissenschaftliche Herausforderung. In: Jürgen Wilke (Hrsg.): Ethik der Massenmedien. Wien: Braumüller: 144-157.

Bentele, Günter (1997): Grundlagen der Public Relations. Positionsbestimmung und einige Thesen. In: Wolfgang Donsbach (Hrsg.): Public Relations in Theorie und Praxis. Grundlagen und Arbeitsweise der Öffentlichkeitsarbeit in verschiedenen Funktionen. München: Reinhard Fischer: 21-36.

Bentele, Günter (1997a): PR-Historiographie und funktional-integrative Schichtung. In: Peter Szyszka (Hrsg.): Auf der Suche nach Identität. PR-Geschichte als Theoriebaustein. Berlin: Vistas: $137-169$.

Bernays, Edward L. (1947): The Engineering of Consent. In: Annals of the American Academy of Political and Social Science, 250, 113-120.

Botan, Carl (1992): Public Relations as a Science. Implications of Cultural Differences and International Events. In: Horst Avenarius, Wolfgang Armbrecht (Hrsg.): Ist Public Relations eine Wissenschaft? Opladen: Westdeutscher Verlag: 17-31.

Cutlip, Scott M.; Center, Allen H.; Broom, Glen M. ( $\left.{ }^{8} 1994\right)$ : Effective Public Relations. Englewood Cliffs: Prentice Hall.

DPRG (Deutsche Public Relations Gesellschaft) (Hrsg.) (1990): Qualifikationsprofil Öffentlichkeitsarbeit/Public Relations. Bonn [Informationsbroschüre].

DPRG (Deutsche Public Relations Gesellschaft) (Hrsg.) (2005): Qualifikationsprofil Öffentlichkeitsarbeit/Public Relations. Bonn [Informationsbroschüre].

24 Definitionen von PR, die von Praktikern stammen, blenden in ihrer Semantik durchweg den Aspekt der Konstruktion, der Manipulation oder der Täuschung aus - weil diese negativ assoziiert sind. Aber auch eine wissenschaftliche Definition darf mit solchen Begriffen ebenfalls nicht hantieren, weil sie wertend wirken. Auch von daher ist der hier vorgeschlagene Begriff „Differenzmanagement“ in jedem Fall vorteilhaft. 
Fischer-Appelt, Bernhard (2005): Die Moses-Methode. Führung zu bahnbrechendem Wandel. Hamburg: Murmann.

Fröhlich, Romy (2005): Die Problematik der PR-Definition(en). In: Günter Bentele, Romy Fröhlich, Peter Szyszka (Hrsg.): Handbuch der Public Relations. Wissenschaftliche Grundlagen und berufliches Handeln. Wiesbaden: Verlag Sozialwissenschaften: 95-109.

Grunig, James E.; Hunt, Todd (1984): Managing Public Relations. Fort Worth.

Haacke, Wilmont (1957): Das Vertrauen der Öffentlichkeit (,Public Relations'), in: Jahrbuch der Absatz- und Verbrauchsforschung, 3: 129-153.

Habermas, Jürgen (1995): Theorie des kommunikativen Handelns. Bd. 1. Frankfurt: Suhrkamp.

Haedrich, Günther; Barthenheier, Günter; Kleinert, Horst (Hrsg.) (1982): Öffentlichkeitsarbeit. Dialog zwischen Institutionen und Gesellschaft. Ein Handbuch. Berlin: De Gruyter.

Harlow, Rex (1976): Building a Public Relations Definition. In: Public Relations Review, 2, Nr. 4: 34-42.

Harlow, Rex (1977): Public Relations Definitions Through the Years. In: Public Relations Review, 3, Nr. 2: 49-63.

Hempel, Carl Gustav (1974): Grundzüge der Begriffsbildung in der empirischen Wissenschaft. Düsseldorf: Bertelsmann.

Kauffman, Louis H. (1987): Self-Reference and Recursive Forms. In: Journal of Sociological and Biological Structure, 10: 53-72.

Kunczik, Michael (2002): Public Relations. Konzepte und Theorien. Köln/Weimar/Wien: Böhlau. Lentz, Ingo (1978): Die Öffentlichkeitsarbeit der Spitzenverbände. Düsseldorf/Wien: Econ.

L'Etang, Jacquie; Pieczka, Magda (eds.) (2006): Public Relations. Critical Debates and Contemporary Practice. Mahwah/London: Lawrence Erlbaum.

Luhmann, Niklas (1970): Soziologische Aufklärung. Opladen: Westdeutscher Verlag.

Luhmann, Niklas (2003): Einführung in die Systemtheorie. Darmstadt: Wiss. Buchgesellschaft.

Maser, Siegfried (1971): Grundlagen der allgemeinen Kommunikationstheorie. Berlin.

Meffert, Heribert ('2000): Marketing. Grundlagen marktorientierter Unternehmensführung. Konzepte - Instrumente - Praxisbeispiele. Wiesbaden: Gabler.

Merten, Klaus (1977): Kommunikation. Eine Begriffs- und Prozeßanalyse. Opladen. Westdeutscher Verlag.

Merten, Klaus (2005): Zur Ausdifferenzierung der Mediengesellschaft: Wirklichkeitsmanagement als Suche nach Wahrheit. In: Klaus Arnold, Christoph Neuberger (Hrsg.): Alte Medien - Neue Medien. Festschrift für Jan Tonnemacher. Wiesbaden: VS Verlag für Sozialwissenschaften: 21-39.

Merten, Klaus (2007): Medienanalyse in der Mediengesellschaft. In: Thomas Wägenbaur (Hrsg.): Medienanalyse. Methoden, Ergebnisse, Grenzen. Baden-Baden: Nomos: 21-50.

Merten, Klaus (2008): Die Anfänge des Kommunikationsmanagement: Mose in der Wüste. In: Klaus Merten, Elke Neujahr (Hrsg.): Handbuch der Unternehmenskommunikation 2008. Köln: Von Halem (im Druck).

Merten, Klaus; Westerbarkey, Joachim (1994): Public Opinion und Public Relations. In: Klaus Merten, Siegfried J. Schmidt, Siegfried Weischenberg (Hrsg.): Die Wirklichkeit der Medien. Opladen: Westdeutscher Verlag: 188-211.

Merton, Robert King (1967): Funktionale Analyse. In: Heinz Hartmann (Hrsg.): Moderne Amerikanische Soziologie. Stuttgart: Enke: 119-150.

Mintzberg, Henry ( $\left.{ }^{2} 1980\right)$ : The Nature of Managerial Work. Englewood Cliffs: Prentice Hall.

Noelle-Neumann, Elisabeth; Schulz, Winfried; Wilke, Jürgen (Hrsg.) (51999): Fischer Lexikon Publizistik/Massenkommunikation. Frankfurt am Main: Fischer.

Oeckl, Albert (1964): Handbuch der Public Relations. Theorie und Praxis in Deutschland und der Welt. München: Süddeutscher Verlag.

Oelert, Jochen (2003): Internes Kommunikationsmanagement. Rahmenfaktoren, Gestaltungsansätze und Aufgabenfelder. Wiesbaden: Gabler.

Parsons, Talcott (1959): General Theory in Sociology. In: Robert King Merton, Leonard Broom, Leonard S. Cottrell Jr. (Hrsg.): Sociology Today. Problems and Prospects. New York: Harper, Bd. I: 3-38. 
Pavlik, John V. (1987): Public Relations. What Research Tells Us. Newbury Park: Sage.

Posner-Landsch, Marlene (2007): StoryTelling - StorySelling. Märchen und Märchenerzähler in der Wirtschaft. Köln: von Halem.

Röttger, Ulrike (2000): Public Relations - Organisation und Profession. Opladen: Westdeutscher Verlag.

Rühl, Manfred (1992): Public Relations ist, was Public Relations tut. Fünf Schwierigkeiten, eine allgemeine PR-Theorie zu entwerfen. In: prmagazin, 23, Nr. 4: 35-46.

Scharf, Wilfried (1971): ,Public relations' in der Bundesrepublik Deutschland. In: Publizistik, 6, Heft 2: $163-180$.

Schmidt, Siegfried J. (1995): Werbung, Medien und Kultur. Opladen: Westdeutscher Verlag.

Shands, Harley C. (1967): Novelty as Object: Precis for a General Psychological Theory. In: Archives of General Psychiatry, 17: 1-4.

Stieler, Caspar (21969): Zeitungs Lust und Nutz. Bremen: Schünemann.

Watzlawick, Paul/Beavin, Janet H./Jackson, Don D. ('21971): Menschliche Kommunikation. Formen, Störungen, Paradoxien. Bern, Stuttgart, Wien: Huber.

Weber, Stefan (2005): Non-dualistische Medientheorie. Konstanz: UVK.

Westerbarkey, Jochen (1998): Das Geheimnis. Leipzig: Kipenheuer.

Wienand, Edith (2003): Public Relations als Beruf. Opladen: Westdeutscher Verlag.

Zedtwitz-Arnim, Georg-Volkmar Graf von (1961): Tu Gutes und rede darüber. Public Relations für die Wirtschaft. Berlin: Ullstein (21982).

\section{Perspektiven für eine europäische Öffentlichkeit?}

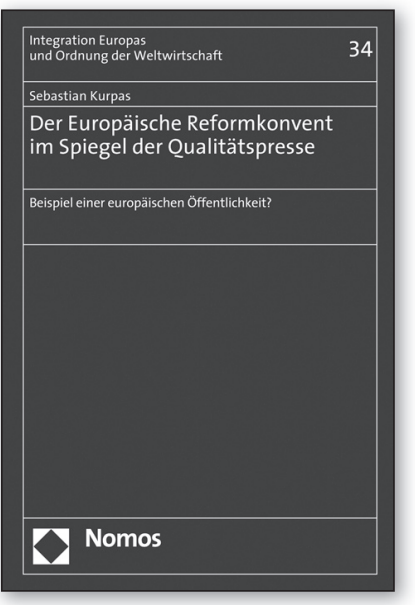

\section{Der Europäische Reformkonvent im Spiegel der Qualitätspresse}

Beispiel einer europäischen

Öffentlichkeit?

Von Dr. Sebastian Kurpas

2008, 230 S., geb., 48,- $€$,

ISBN 978-3-8329-3370-8

(Integration Europas und Ordnung

der Weltwirtschaft, Bd. 34)

Bitte bestellen Sie bei Ihrer Buchhandlung oder bei Nomos | Telefon 07221/2104-37 | Fax -43 |

www.nomos.de | sabine.horn@nomos.de

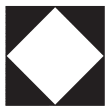

Nomos 